\title{
'Paul Singer \\ Linksintellektuelle in Brasilien: \\ Die Erfahrung mit der Macht
}

Zusammenfassung: Mit dem Übergang zur Demokratie nach 20 Jahren Militärdiktatur zwischen 1964 und 1984 steigen oppositionelle Intellektuelle in Positionen der Macht auf. Bezeichnenderweise sind es vor allem Ökonomen, die in hohe Beraterpositionen oder gar in verantwortliche Regierungsämter aufrücken, und dies unter einem Präsidenten Sarney, der vor der „Öffnung " des Militärregimes einer seiner zivilen Repräsentanten war. Die »Ökonomen des $P M D B \ll$ (der ehemals oppositionellen, nun aber größten Regierungspartei) machen Furore mit einem Plan zur Bekämpfung der Inflation, deren Ursache sie in »inertialen «, d.h. in Trägheitsmomenten, die sich ungebrochen fortsetzen, ausmachen. Ein "heterodoxer Schock « eingefrorener Preise soll der Inflation die Grundlage ein für alle Mal entziehen. Die Massen, der ständigen Preissteigerung müde, sind zunächst begeistert. Doch der Plan ist erfolglos, die Inflation kehrt mit höheren Zuwachsraten als zuvor zurück. Die linksintellektuellen Ökonomen haben die Inflation eben nur technokratisch bekämpft und nicht begreifen können, daß eine Änderung der Regeln des wirtschaftspolitischen Prozesses insgesamt notwendig gewesen wäre, um die Erfordernisse der Akkumulation und die Hoffnungen der Massen auf eine Verbesserung ihrer Lebensbedingungen miteinander zu vereinbaren. Die linken »Ökonomen des PMDB scheitern an ihrer eigenen »déformation professionelle".

\section{Der Hintergrund}

Während der langen Jahre des Militärregimes von 1964 bis 1985 begannen die brasilianischen Intellektuellen eine höchst bedeutsame Rolle im politischen Leben zu spielen, als die »normalen Kanäle« der Opposition - Parteien, Gewerkschaften, Presse - durch die Repression weitgehend verstopft waren. Intellektuelle waren in bestimmten Grenzen geschützt, vielleicht weil man annahm, sie kreisten mit ihren Aktivitäten um sich selbst und sie seien folglich unfähig, die Massen zu beeinflussen. Die notorische Unverständlichkeit des intellektuellen Diskurses machte ihn harmlos; die gerade errichtete Ordnung lief durch ihn keine Gefahr. In Wirklichkeit war es allerdings nicht ganz so. Die vermeintlich »technischen « Ansichten der oppositionellen Intellektuellen, vor allem der Ökonomen, konnten häufig von den Massenmedien verbreitet werden; dort wurden sie von den Journalisten in Umgangssprache »übersetzt «. Damit war dafür gesorgt, daß sie einen größtmöglichen Widerhall fanden. 
Doch um das Auftauchen der Intellektuellen als politisch gewichtigen Faktor in Brasilien ganz zu verstehen, muß man einige strukturelle Aspekte dieses Prozesses beruicksichtigen. Hierbei handelt es sich in erster Linie um den beschleunigten Ausbau der Universitäten, der in Brasilien als Krönung der nachgerade explosionsartigen Ausdehnung des Schulsystems in den 50er und 60er Jahren stattfand. Der relative Schulbesuch der jeweiligen Altersgruppen der Bevölkerung stieg rapide an und provozierte eine regelrechte Überschwemmung des höheren Schulwesens. In wenigen Jahren wurden in allen Bundesstaaten staatliche Universitäten gegründet, abgesehen von den verschiedenen konfessionellen, v.a. katholischen Universitäten. Aber die Nachfrage nach Schul- und Studienplätzen übertraf dies alles und hatte bereits während des Militärregimes in kurzer Frist eine Vervielfachung der Zahl privater Schulen und Hochschulen zur Folge.

Der Ausbau der Universitäten öffnete für Intellektuelle einen weiten Arbeitsmarkt. Damit ging eine quantitative Ausdehnung der Zahl der Intellektuellen ebenso einher wie eine Veränderung ihrer sozialen Herkunft und ihrer ideologischen Ausrichtung. Zuvor gab es nur eine beschränkte Elite, größtenteils an den juristischen Fakultäten herangewachsen, aus den herrschenden Klassen stammend und in aller Regel konservativ. Dann aber vollzog sich mit der zahlenmäßigen Zunahme der Intellektuellenschicht - die nun zum größten Teil in den sogenannten »Volksschichten « (classes populares) rekrutiert wurde - eine Diversifizierung ihrer Fachrichtungen. Der typische Intellektuelle war nun nicht mehr Jurist, sondern Soziologe, Politologe, Ökonom, auch Physiker, Mediziner, Geologe etc. Als öffentliche Figur erschien der Intellektuelle immer mehr als »Spezialist«, anerkannter Fachmann auf einem bestimmten Gebiet. Und in politischer Hinsicht wandten sich die Intellektuellen zur Linken.

Natürlich gab es bereits vor der Universitäts-Expansion linke Intellektuelle in Brasilien. Aber im allgemeinen standen sie am Rande des »establishments «, außerhalb der Fakultäten und Akademien. Nach der Reform jedoch konnte man das intellektuelle »Establishment « hauptsächlich in den Universitäten vorfinden und dort hatten die Intellektuellen von Anfang an eine herausragende Stellung. Zu Beginn des Militärregimes war die Linke im intellektuellen Leben insgesamt vorherrschend und dies nicht nur an den Universitäten, sondern auch in den Redaktionen, in Theater, Kino und in der populären Musik.

Diese Linksintellektuellen waren es, die nach Beginn der Militärdiktatur als Oppositionelle und Kritiker des Militärregimes hervortraten. 1969 säuberte die Regierung die staatlichen Universitäten: viele Professoren, fast alle mit ausgezeichnetem Ruf, wurden so entfernt. Eine kleine Gruppe der aus den Universitäten vertriebenen Professoren gründete daraufhin das CEBRAP (Brasilianisches Zentrum für Analyse und Planung) ${ }^{*}$, das schnell zu einem wichtigen Zentrum mit großer Ausstrahlungs-

* Mitarbeiter des CEBRAP waren neben Paul Singer auch Fernando Henrique Cardoso, Otavio Ianni, José Arthur Gianotti, um nur einige Hochschullehrer mit internationalem Ruf zu nennen. - Anm. der Redaktion 
kraft wurde. Von ihm gingen Ideen aus, in ihm wurden Theorien, Analysen und Datenmaterial ausgearbeitet, die die oppositionellen Aktivitäten an den Universitäten, in der Presse, in den kirchlichen Basisgemeinden und später, als es zur »politischen Öffnung « kam, auch in den Gewerkschaften und Oppositionsparteien (bis 1979 gab es nur eine Oppositionspartei) anregten. Im Jahre 1972 wurde das Wochenblatt »Opiniao« (Meinung) gegründet, in dessen Spalten viele Intellektuelle trotz der Zensur Informationen, Analysen und theoretische Ansätze veröffentlichten, die unter den Studenten und in der sogenannten »öffentlichen Meinung « enorme Verbreitung fanden. In den folgenden Jahren wurden andere unabhängige Forschungsinstitute gegründet (auch in Nachbarländern), die ansehnliche Forschungsarbeiten und theoretische Reflektionen hervorbrachten; fast alle waren regimekritisch. Nach und nach erschienen noch andere Wochenzeitungen, die insgesamt die sogenannte »Alternativpresse « bildeten. Diese verschwand erst wieder, als auch die großen Zeitungen sich der Zensur nicht mehr beugten und nach und nach ihre Spalten den Intellektuellen öffneten, die bis dahin nur in den unabhängigen Zentren und der Alternativpresse hatten wirken können. Mit der »Öffnung « in der zweiten Hälfte der siebziger Jahre gingen die politischen Aktivitäten wieder von den Universitäten aus und viele wurden damit zu wichtigen Stützpunkten im Kampf für die Demokratie.

\section{Die Ideologie der Linksintellektuellen}

Angesichts der ideologischen Vielfalt der Linksintellektuellen in Brasilien ist es schwierig, dieses Thema ohne riskante Verallgemeinerungen zu behandeln. Es ist aber auch unmöglich, über die Linksintellektuellen zu diskutieren, ohne dieses Thema anzuschneiden, einfach weil der Terminus »links « viel zu vage und unpräzis geworden ist. Es gibt eine Grauzone der Undefiniertheit zwischen progressivem Liberalismus und Sozialismus, in der ein Großteil der Intellektuellen sich ständig vor- und rückwärts bewegt. Als Ausgangspunkt kann man unterstellen, daß die Mehrheit der brasilianischen Intellektuellen eine kritische Haltung gegenüber dem peripheren Kapitalismus Brasiliens einnimmt. Die Kritik richtet sich hauptsächlich gegen die ökonomische Ungleichheit, die die Entwicklung selbst verstärkt. Im Verlauf des schnellen Wirtschaftswachstums seit dem Ende des 2. Weltkrieges entwikkelte sich Brasilien zu einer Art Weltmeister der Ungleichheit. Während des Militärregimes wurden darüber Daten bekannt (die Frage nach dem »Einkommen« wurde zum ersten Mal im demographischen Zensus 1960 erhoben) und diese wurden als Hauptargument gegen die Wirtschafts- und Sozialpolitik der damaligen Regierung verwendet.

Die Frage ist natürlich, ob die tendenzielle Vergrößerung der Ungleichheit dem Kapitalismus innewohnt oder ob sie durch institutionelle Veränderungen im Kapitalismus umgekehrt werden kann. Traditionell kreiste diese Debatte um die Frage der $\gg$ Reife« des Landes für die sozialistische Revolution, oder um den Charak- 
ter - ob sozialistisch oder nicht - einer »brasilianischen Revolution « unter der nicht hinterfragten Annahme, es müsse auf jeden Fall eine Revolution geben. Diese Debatte erstarb, als der Versuch des bewaffneten Kampfes gegen die Militärdiktuatur mit einem totalen Mißerfolg endete. Viele junge Intellektuelle engagierten sich in diesem Versuch. Der Kampf dauerte von 1969 bis Mitte 1972 ca. dreieinhalb Jahre (ohne den »Guerillakrieg von Araguaia« zu zählen, der 1972-74 geführt wurde). Der Sieg der Repression wurde im wesentlichen deshalb möglich, weil jegliche Unterstützung durch die Arbeiterklasse fehlte. Nach dieser Erfahrung engagierte sich der größte Teil der Linken im politischen Kampf gegen die Diktatur, gezwungen von der Überzeugung, daß der Sozialismus - wenigstens in diesem Stadium - nicht auf der Tagesordnung stünde. Das Hauptziel war der Sturz der Diktatur und die Wiederherstellung der Demokratie im Land.

Die Frustrationen mit dem »realen Sozialismus« trugen auch dazu bei, daß die sozialistische Alternative in der intellektuellen Debatte keine Rolle mehr spielte. Die Invasion in der Tschechoslowakei 1968 und die wiederholten Repressionen gegen Arbeiterstreiks in Polen 1970, 1976 und 1981 wirkten wie eine kalte Dusche auf den Enthusiasmus der brasilianischen Intellektuellen über eine Revolution von Arbeitern und Bauern. Natürlich wurde auch aus der Stalinismuskritik im Lager der Linken das Projekt eines demokratischen Sozialismus abgeleitet, der zwar die Klassen beseitigen sollte, ohne aber die politischen Rechte und die bürgerlichen Freiheiten abzuschaffen, die im Kapitalismus erobert worden waren. Aber die Tatsache, daß dieses Projekt nirgendwo konkretisiert wurde und auch der chilenische Versuch Allendes vereitelt wurde, machte diese Option ziemlich abstrakt. Auch die wiederholten Regierungen der europäischen Sozialdemokratie, die ohne irgendwelche handgreiflichen Fortschritte in Richtung auf einen neuen Typ des Sozialismus blieben, führten in den Köpfen der meisten kritischen Intellektuellen zur Verdrängung der sozialistischen Alternative.

Mit der politischen Öffnung und den wachsenden ökonomischen Schwierigkeiten, hervorgerufen durch die Verschlechterung der internationalen Konjunktur in der zweiten Hälfte der 70er Jahre, wandte sich auch ein größerer Teil der ökonomischen Eliten der Opposition zu. Dadurch wurde eine organische Verbindung zwischen Linksintellektuellen, hauptsächlich Wirtschaftswissenschaftlern, und jenem Teil der Bourgeoisie begünstigt, der nationale und demokratische Positionen einnahm. Die immer entschiedenere Beteiligung dieser Fraktion am Kampf gegen das Militärregime verstärkte bei den Linksintellektuellen die Tendenz, die Dichotomie zwischen Kapitalismus und Sozialismus durch den Gegensatz von »modern « und »archaisch« zu ersetzen. Der Kampf ginge nicht mehr um die Abschaffung des Kapitalismus in Brasilien, sondern um seine Modernisierung, um die Auslöschung seiner anachronistischen Facetten, die ihn »wild « und autoritär machten und die starke Konzentration der Einkommen hervorbrachten.

Doch wurden diese Fragen nicht in öffentlicher Debatte erörtert. Der Übergang des größten Teils der Intellektuellen von einer sozialistischen zu einer demokratisch- 
modernistischen oder liberal-progressiven Einstellung vollzog sich vielmehr stillschweigend. Die Spaltung zwischen denjenigen, die ihre politische Haltung änderten und denen, die es nicht taten, zeigte sich erst offen, als 1979/80 Parteien zugelassen wurden und statt nur einer Oppositionspartei nun drei Parteien entstanden: der PMDB, der den Charakter einer breiten Front des Widerstandes gegen das Militärregime beibehielt; der PT, der aus der Gewerkschaftsbewegung und dem radikalsten Flügel der katholischen Kirche entstand; und der PDT, gegründet von Leonel Brizola mit dem Ziel, den alten PTB unter seiner Führung wieder auferstehen zu lassen".

Der PMDB proklamierte sich als Partei »über den Klassen« (»interklassistisch«), mit einem demokratischen Erneuerungsprogramm und einem Programm sozialer Reformen in den Grenzen des Kapitalismus. Der PT wies sich als Partei der Arbeiterklassen aus, die sich dem Aufbau einer Gesellschaft »ohne Ausgebeutete und ohne Ausbeuter « verschrieb. Und der PDT versuchte, das populistische Erbe eines Getulio Vargas (G. Vargas, brasilianischer Präsident von 1930-1945 und von 19501954) wiederzubeleben. Als Ziel definierte die Partei einen »sozialismo moreno« (Sozialismus mit dunkler Hauptfarbe), eine speziell brasilianische Version der sozialdemokratischen Internationale.

Der Großteil der Intellektuellen, der einer wie auch immer gearteten sozialistischen Perspektive treu blieb, trat dem PT bei. Ein weitaus kleinerer Teil ging zum PMDB und zum PDT. Die »modernistischen « Intellektuellen ihrerseits gingen zum größten Teil in den PMDB. In einer empirischen Untersuchung (durchgeführt von Martins Rodrigues) wurden die Abgeordneten des Parlaments in Liberale, Sozialdemokraten, moderate Sozialisten und extreme Sozialisten klassifiziert. Von den »Sozialdemokraten «, die den von uns als »modernistisch « bezeichneten Demokraten entsprechen, sind $67 \%$ beim PMDB, 4,7\% beim PDT und nur $0,6 \%$ beim PT und anderen traditionellen Linksparteien. Die »moderaten Sozialisten « gehören in ihrer Mehrzahl $(67,7 \%)$ ebenfalls zum PMDB, $12,3 \%$ sind im PDT und $10,8 \%$ im PT und anderen traditionellen Linksparteien. Nun, die »extremen Sozialisten« sind zu 56\% im PT und anderen traditionellen Linksparteien, 28\% befinden sich im PMDB und $16 \%$ im PDT.

Insgesamt sind also 63\% der sozialdemokratischen und sozialistischen Abgeordneten - abgesehen davon, daß Teile anderen politischen Vereinigungen oder Gruppen,

* Der PMDB (Partei der demokratischen Bewegung Brasiliens) ist aus der einzigen unter dem Militärregime zugelassenen Partei der Opposition hervorgegangen. Der PT (Partei der Arbeiter) entstand als Folge der Streikbewegung insbesondere in Sao Paulo gegen Ende der 70er Jahre. Der PDT (Demokratische Arbeiterpartei) knüpft an die »populistischen Traditionen des PTB (Brasilianische Arbeiterpartei) aus der Zeit vor dem Militärregime an. Andere Parteien, die im Text erwähnt werden, sind: der PCB (Brasilianische Kommunistische Partei, eurokommunistisch orientiert), der PC do B (Kommunistische Partei Brasiliens, eher marxistisch-leninistisch-maoistisch orientiert), PSB (Brasilianische Sozialistische Partei). Der PFL (Partei der Liberalen Front) ist eine Abspaltung der Partei der Militärs PDS (Sozialdemokratische Partei) und ist als kleinerer Koalitionspartner mit an der Regierung beteiligt. - Anm. der Redaktion 
die hier nicht berücksichtigt wurden, zusätzlich angehören - im PMDB, 8,4\% im PT und anderen traditionellen Linksparteien und 7,7\% im PDT. Nach der erwähnten Studie repräsentiert dieser Block 60\% des Abgeordnetenhauses. Diese Ergebnisse lassen keinen Zweifel darüber, daß die Mehrheit der brasilianischen Linken heute im PMDB ist, daß sich aber die Mehrheit der sozialistischen Linken (nur 5,7\% der Linken im Abgeordentenhaus) im PT, PCB, PC do B und PSB befindet. So etwas Ähnliches ist wohl auch bei den Linksintellektuellen passiert: die demokratisch-modernistische Mehrheit ist im PMDB, die sozialistische Minderheit befindet sich vor allem im PT.

Auch wenn all dies eine offensichtliche Vereinfachung des ideologischen Panoramas ist, dient es doch der Hervorhebung von zwei wesentlichen Faktoren der nachfolgenden Diskussion: Erstens gab es eine Standortverschiebung bei einem Großteil der Linken, einschließlich der Intellektuellen, hin zur Akzeptanz des Kapitalismus als institutionellem Rahmen, innerhalb dessen versucht wird, Lösungen für die brasilianische Problematik der extrem ungleichen Einkommensverteilung zu finden. Zweitens hat sich die Linke mit dieser Position mehrheitlich im PMDB organisiert. Seit März 1985 (also nach dem Ende des Militärregimes und der Errichtung der »Neuen Republik « Anm. der Redaktion) ist der PMDB an der Macht beteiligt. Bei den letzten Wahlen erreichte diese Partei eine breite Mehrheit. Er stellt (bis auf einen) alle Gouverneure der Bundesstaaten und hält die absolute Mehrheit der Abgeordneten in der Verfassungsgebenden Versammlung (Constituinte) ${ }^{*}$.

\section{Intellektuelle an der Macht}

Der sehnlich erwartete Sturz des Militärregimes ereignete sich sehr brasilianisch unter festlicher Begleitmusik, im Zuge einer nie klar ausgesprochenen Übereinkunft zwischen den Militärs, die sich freiwillig von der Bühne zurückzogen, und einer Koalition der zwei größten Parteien PMDB und PFL: Im März 1985 wurde die neue Zivilregierung nach indirekter Wahl mit José Sarney als Präsidenten eingesetzt. Dieser, obwohl nur amtierender Vizepräsident, wurde wegen der Kranheit und des sehr schnell folgenden Todes des designierten Präsidenten Tancredo Neves zum Präsidenten gemacht. Sarney war bis zur Spaltung im Juli 1984 Vorsitzender des PDS und einer der zivilen Exponenten des Militärregimes gewesen. Sein Kabinett war von Tancredo gebildet worden und bestand aus einer Mehrheit von PMDB- und einer Minderheit von PFL-Ministern.

Linksintellektuelle waren auf einmal mit dem Eintritt des PMDB in die Regierung an der Macht beteiligt. Eine Reihe von Linksintellektuellen ist auch als Abgeordnete

* Im November 1986 wurden auch die Repräsentanten für eine Verfassungsgebende Versammlung gewählt, deren Auftrag es war, bis zum November 1987 eine neue Verfassung auszuarbeiten, die diejenige des Militärregimes ersetzen sollte. Die Arbeiten an der neuen Verfassung haben sich allerdings verzögert. - Anm. der Redaktion 
ins Parlament oder in die Verfassungsgebende Versammlung gewählt worden. Doch waren das nur sehr wenige, obwohl von großer Prominenz wie Fernando Henrique Cardoso, der bekannteste von ihnen, Führer des PMDB im Senat, oder die Abgeordneten Florestan Fernandes (PT), der »Vater der brasilianischen Soziologie «, und der Ökonom José Serra (PMDB). Die Präsenz der Linksintellektuellen in der Verfassungsgebenden Versammlung ist im Verhältnis sehr klein und kann nur mit Einschränkungen als Ausübung von Macht bezeichnet werden. Solche Machtausübung jedoch gab es in der Exekutive und dort auf sehr spezifische Weise: durch die sogenannten »Ökonomen des $\mathrm{PMDB}$ «, die in die Regierung eintraten und dort mit großer Autorität und Verantwortung ausgestattet waren.

Im ersten Kabinett Sarney besetzte der Ökonomieprofessor Joao Sayad das Planungsministerium und zog eine Gruppe von Kollegen in die Regierung nach. Bei der ersten wichtigen Regierungsneubildung im August 1985 wurde der Unternehmer Dilson Funaro zum Finanzminister berufen, der zu seiner direkten Unterstützung die Ökonomen Cardoso de Melo und Gonzaga Belluzzo mitbrachte. Ins Direktorium der Zentralbank Brasiliens und der Bank von Brasilien rückten weitere »Ökonomen des PMDB «, wie Pérsio Arida, André Lara Rezende, Adoraldo Moura etc. auf. Sie alle sind berühmte Dozenten der besten brasilianischen Universitäten, fast alle haben in den USA promoviert.

Es ist interessant zu bemerken, daß unter den zahlreichen Linksintellektuellen ausschließlich Ökonomen in die Regierung aufgenommen wurden und sogar Posten erhielten, die nicht zu ihrem Fachgebiet gehören. Z.B. wurde Celso Furtado, der renommierteste der »kritischen Ökonomen «, im Februar 1986 zum Kultusminister ernannt. Die linken Juristen, Mediziner, Physiker, Politologen etc., die auch dem PMDB angehörten, blieben außen vor oder wurden bestenfalls von der Regierung beauftragt, Studien und Pläne zu erarbeiten. Die Juristen, Mediziner etc., die in die Regierung eintraten, waren mit wenigen Ausnahmen keine Linken, und sie wurden nur auf Posten in der zweiten Reihe gesetzt.

Es ist kaum zu glauben, daß dies reiner Zufall gewesen ist. Es scheint etwas zu geben, was die Ökonomen von den meisten auf Modernisierung und Demokratisierung orientierten Intellektuellen unterscheidet. Die Ökonomen akzeptieren den Kapitalismus als institutionellen Rahmen, in dem man politische Maßnahmen formulieren kann, nehmen also die »Marktgesetze « ernst. Letztlich bedeutet dies, daß die Interessen der kapitalischen Klasse als hegemoniale Interessen heute und immerdar behandelt werden, wie sehr sie sich auch wünschen mögen, den Interessen der Arbeiter oder der Armen Priorität einräumen zu können. Durch seine theoretische Ausbildung und Praxis »weiß« der Ökonom, daß die Produktivkräfte nur entsprechend dem Profitprinzip bewegt werden können und daß deshalb die Ziele der Wirtschaftspolitik nur im Einklang mit den Zielsetzungen des Kapitals erreicht werden können. Die meisten modernistisch-demokratischen Intellektuellen sind sich kaum dieser » Tatsache « bewußt, oder, wenn sie es sind, richten sie ihre politischen Vorschläge nicht danach. Auf anderen Gebieten - gesetzgeberisch-institutionell, in der 
Technologie, im Bildungswesen z.B. - erscheinen die Kapitalinteressen gebrochen und untergeordnet, können auf der gleichen Ebene wie die der anderen Klassen berücksichtigt werden. In der Außenpolitik z.B. würde ein modernistischer Demokrat versuchen, nationalistische und Dritte-Welt- bis hin zu antiimperialistischen Positionen zu vertreten, ohne sich sehr um die Möglichkeit der Beeinträchtigung der Auslandsinvestitionen zu kümmern, die er sicherlich nicht als vorrangig ansehen würde. Aber wenn dieser modernistische Demokrat Ökonom wäre, ist es sehr wahrscheinlich, daß er sehr genau auf die Rück wirkungen der verschiedenen politischen Alternativen auf die Kapitalbewegungen achten würde; in der Praxis ist er eben den Interessen der herrschenden Klasse sehr viel näher als Intellektuelle mit anderer Qualifikation.

Diese Argumente begründen offensichtlich nicht, warum T. Neves und J. Sarney den „Ökonomen des PMDB« bewußt die Türen zur Macht geöffnet haben, während sie sie für die anderen progressiven Intellektuellen der gleichen Partei verschlossen hielten. Die unabweisliche Tatsache ist, daß sie es taten, und ich bin überzeugt, daß dies etwas mit der Besonderheit des Ökonomen zu tun hat. Tancredo Neves hatte tasächlich keinerlei Intention, irgendeinen Teilbereich wirtschaftspolitischer Macht Joao Sayad und seinen progressiven Beratern anzuvertrauen. Tancredo war in dieser Hinsicht durch und durch konservativ und beabsichtigte, die Wirtschaftspolitik von einer rechten Ökonomengruppe unter der Regie seines Neffen Francisco Dornelles machen zu lassen, dem er das Finanzministerium anvertraute. Aber der Tod vereitelte seine Absicht. Sarney zeigte sich als Präsident weniger konservativ und hörte auf Sayad, der sich entschieden Dornelles Wirtschaftspolitik widersetzte. Dies ist aus verschiedenen Gründen zu erklären. Sarney ist aus Maranhao, einem Bundesstaat, der in der ärmsten und zurückgebliebensten Ecke des Landes liegt. Eine seiner Prioritäten ist es, die Regionalentwicklung mit großen infrastrukturellen Projekten voranzubringen. Dies aber ist unvereinbar mit einer Politik der Einsparung öffentlicher Mittel und hoher Zinsen, wie sie von Dornelles betrieben wurde. Dornelles wollte die Inflation bekämpfen (die bei $300-400 \%$ im Jahr lag) und ein Abkommen mit dem IWF erreichen. Sayad vertrat die Meinung, daß die gleichen Ziele mit einer Politik der Reduzierung der an die ausländischen Gläubiger zu transferierenden Werte und der Staatsausgaben erreicht werden könnten.

Sayads Position hatte im PMDB viel mehr Unterstützung, und Sarney wiederum war - im Gegensatz zu Tancredo - abhängig von der Unterstützung der Mehrheitspartei. Tancredo als historischer Führer des PMDB hätte der Partei eventuell die Wirtschaftspolitik aufdrücken können, die er für notwendig hielt. Sarney als kürzlicher Überläufer des Militärregimes mußte das Vertrauen des PMDB erobern und deshalb eine Konfrontation mit dessen Führung vermeiden. Folglich ließ er Dornelles Rücktritt im August 1985 zu und nominierte an seiner Stelle den Industriellen Dilson Funaro aus Sao Paulo, der auf der gleichen Linie wie Sayad und wie andere »Ökonomen des $\mathrm{PMDB} \ll$ lag. 


\section{Die Erfahrung der Macht}

\section{Theoretische Vorbereitung}

Im zweiten Halbjahr 1984, als der Übergang zu einer Zivilregierung unter Präsident Tancredo Neves gesichert war, begannen zwei junge Ökonomieprofessoren der Katholischen Universität von Rio de Janeiro (PUC-Rio), André Lara Rezende und Pérsio Arida, sich mit der scheinbar entscheidenden ökonomischen Frage zu beschäftigen, wie man der Inflation Herr werden könne. Die traditionell in der lateinamerikanischen Linken vorherrschende Theorie der Inflation war »strukturalistisch«: als Hauptgrund des Phänomens wurde die Unelastizität des Güterangebots infolge der Starrheit der ererbten Kolonialstrukturen ausgemacht. Die ungenügende Produktion von Lebensmitteln ist durch den Großgrundbesitz hervorgerufen, der der breiten Masse der landarbeitenden Bevölkerung den Zugang zum Land verwehre. Das Angebot von Importprodukten ist begrenzt infolge der äußeren Restriktionen, im aktuellen brasilianischen Fall infolge der Krise der Außenverschuldung.

Die strukturalistischen Thesen, die die Notwendigkeit von »strukturellen Reformen « aufzeigten, um die Inflation zu bekämpfen, wurden von den »Ökonomen des PMDB « nicht akzeptiert, obwohl sie den Diskurs der nicht-ökonomischen Linken inner- und außerhalb des PMDB beeinflußten. Die starke Steigerung der brasilianischen Agrarproduktion in den 70er Jahren widerlegte die These, daß die Konzentration des Großgrundbesitzes ein Hindernis für die Ausdehnung des Lebensmittelangebots sei. Wenn die ärmsten Schichten weiterhin unterernährt blieben, sei dies vielmehr auf ihr zu geringes Einkommen zum Kauf von ausreichenden Mengen von Gütern und Dienstleistungen zur Befriedigung der Grundbedürfnisse zurückzuführen. Auch die außenwirtschaftliche Strangulierung erschien angesichts von Importsubstitution und Diversifikation der Exporte weniger Bedeutung als unterstellt zu haben. 1984 hatte die brasilianische Wirtschaft die Rezession überwunden und mit enormen positiven Salden in der Handelsbilanz genug Devisen, um die Zinsen auf die Außenschuld vollständig zu zahlen.

Lara Rezende und Arida entwickelten einen neuen Ansatz zur Erklärung der Inflation, der als »neostrukturalistisch « bekannt wurde. Ihre Grundthese ist, daß in einem Land wie Brasilien mit hoher und chronischer Inflation zwei Ursachen zu unterscheiden sind, nämlich die Indexierung und die Schocks. Die Indexierung ist eine Folge permanenter Inflation. Es werden automatische Anpassungen der finanziellen Aktiv- (und Passiv-)posten sowie der Wechselkurse, der Löhne, der Mieten und vieler staatlich kontrollierter Preise regelrecht instututionalisiert. Die Anpassung entspricht der vorhergegangenen Inflationsrate, gemessen durch irgendeinen offiziellen Index. Wenn die Inflation eine bestimmte Höhe erreicht hat, neigt die Indexierung dazu, sie zu reproduzieren, auch wenn es keine äußeren Schocks oder Steigerungen der Nachfrage und/oder Minderungen des Angebots gibt, die die Preise nach oben treiben. Die Inflation habe demzufolge zwei Komponenten: das ihr innewohnende »Trägheitsmoment« (»inertiale Inflation«), das ein unmittelbares Er- 
gebnis der Indexierung ist, und die durch eventuelle äußere Schocks bewirkten Inflationsanstöße. Die interne Wirkung des externen Schocks ist die Erhöhung der Inflationsrate, während die Indexierung sie auf dem einmal erreichten Niveau hält, also das Verschwinden der Inflation verhindert.

Lara Rezende und Arida kamen zu der Diagnose, daß die aktuelle Inflation in Brasilien ausschließlich »inertiale« Ursachen hatte, da die Wirkungen der äußeren Schocks der Ölpreissteigerung und der Anpassungen an die Schuldenkrise bereits verarbeitet waren. Staatshaushalt und Zahlungsbilanz waren ausgeglichen, doch die »inertiale « Komponente erlaubte es nicht, daß die Inflation unter das Niveau von $200 \%$ jährlich sank. Da eine so hohe Inflationsrate eine ernste Gefahr des Übergangs in eine Hyperinflation darstellt, wenn sie durch irgendeinen neuen Schock beschleunigt wird, war die Eliminierung der »inertialen « Komponente notwendig. Dies sollte mit einer neuen Währung geschehen, deren Wert mit der Inflation der alten Währung, die für einige Zeit weiter zirkulieren sollte, indexiert würde. Gerechnet in der neuen Währung wären alle Werte per Definition konstant; dies würde große Vorteile gegenüber der alten Währung bieten. In wenigen Monaten würden alle die neue Währung benutzen und die »inertiale« Inflation wäre beseitigt.

Der Vorschlag von Lara Rezende und Arida wurde Ende 1984 verbreitet und provozierte einige Diskussionen. Die Hauptkritik richtete sich gegen die Diagnose, die brasilianische Inflation sei nur »inertial«, also der durch die Indexierung bewirkten Trägheit des Preissystems geschuldet. Einer der Kritiker war Franciso Lopes, ein anderer Ökonomieprofessor der PUC-Rio, der vorschlug, als Methode zur Beseitigung der »inertialen« Inflation den von ihm so genannten »heterodoxen Schock « anzuwenden: Zusammen mit der Währungsreform müßten die Löhne auf ein reales Durchschnittsniveau angehoben werden, das sie in einem bestimmten Zeitraum vor der Anwendung des Schocks gehabt hatten. Dasselbe müßte auch mit den übrigen Einkommen geschehen, die bereits (als Kosten) in die Preise eingegangen waren. In die neue Währung konvertiert müßten sie dann eingefroren werden.

Francisco Lopes Vorschlag lag die Überlegung zugrunde, daß bei einer relativ konstanten Inflationsrate (die Konstanz charakterisiert das inertiale Moment) jede Klasse und jede Klassenfraktion ein variables Realeinkommen wegen der Zeitspanne zwischen den nominalen Angleichungen der Einkommen an die Inflation hat. Mit dem Moment der ersten Angleichung erreicht das fragliche Einkommen in realen Zahlen seinen höchsten Wert, sagen wir 100. Danach wird es nach und nach von der Inflation aufgezehrt. Wenn das Einkommen alle drei Monate angeglichen würde und die monatliche Inflation 10\% betrüge, wäre sein realer Wert: 100 im ersten Monat, 90,9 im zweiten Monat und 82,6 im 3. Monat. In diesem Trimester wäre der durchschnittliche reale Wert des Einkommens 91,2 gewesen. Im 4. Monat würde das Einkommen nach der Angleichung wieder in realen Zahlen 100 wert sein. Für den Einkommensbezieher ist es gleich, ob er im Verlauf eines Trimesters jeden Monat 91,9 bezieht oder ein monatliches variables Einkommen von 100, 90,9 und 82,6. Der »heterodoxe Schock« würde jeder Klasse und jeder Klassenfraktion ein Real- 
einkommen verschaffen, das demjenigen äquivalent wäre, das sie durch Inflationsund Indexierungseffekt auch hatte.

Der Vorschlag des »choque heterodoxo « unterstellt wegen der Konstanz der Inflationsrate und der Indexierung aller Preise die Neutralität der Inflation in bezug auf die Einkommensverteilung. Deshalb würde eine Umwandlung aller Einkommen auf ihren realen Durchschnittswert die Eliminierung der Inflation erlauben. Das durch die Inflation erreichte Gleichgewicht würde gehalten, nun aber zu festen Preisen. Die neue Währung wäre nicht an sich wesentlich für den »choque heterodoxo«; sie würde nur die Funktion haben, die inflationistischen Erwartungen zurückzuhalten und den Wirtschaftssubjekten Vertrauen in das Zirkulationsmittel zurückzugeben.

Es muß gesagt werden, daß der »Neostrukturalismus « weit davon entfernt war, einhellige Zustimmung zu ernten, auch nicht bei den »Ökonomen des PMDB«. Einige wiesen die Hypothese zurïck, daß die brasilianische Inflation inertial und neutral in bezug auf die Einkommen sei. Sie wiesen auf die großen Einkommenseinbußen für die Lohnabhängigen hin, die sich 1983 aus den Änderungen der Indexierungsregeln durch die Regierung Figueiredo zur gleichen Zeit ergeben hatten, als die Inflation von einem Niveau um die $100 \%$ auf über $200 \%$ im Jahr anstieg. Es gab tiefe Unzufriedenheit mit dem so gesenkten durchschnittlichen Reallohn; die Unzufriedenheit mit der wirtschaftlichen Situation insgesamt war ohne Zweifel einer der wichtigsten Faktoren für den großen Erfolg der Kampagne für die Direktwahlen (des Präsidenten) in den ersten Monaten 1984. Es war, um das mindeste zu sagen, wenig realistisch, die Einkommensverteilung auf dem Niveau von Ende 1984 und Anfang 1985 stabilisieren zu wollen, wenn die Bevölkerungsmehrheit von dem langersehnten demokratischen Zivilregime nach der Zeit der Diktatur gerade Initiativen einer nachhaltigen Umverteilung der Einkommen erwartete.

Allerdings gewann die »neostrukturalistische« Strömung Anhänger in anderen Ländern, und ihre Vorschläge wurden Mitte 1985 in Argentinien, Israel und Peru in die Praxis umgesetzt. Diese »heterodoxen« Erfahrungen hinterließen eine beachtliche Wirkung in Brasilien, wahrscheinlich ebenso wie auch in anderen Ländern mit hoher und chronischer Inflation. Ein Ideen- und Vorschlagspaket, das vorher nur unter berufsmäßigen Ökonomen zirkulierte, wurde nun vom breiten Publikum diskutiert.

\section{Der Cruzado-Plan}

Ab August 1985 konnten die »Ökonomen des PMDB« die Formulierung und Durchführung der Steuerpolitik, der monetären Belange, der Währungspolitik, der Lohn- und Preispolitik entscheidend beeinflussen. Eine ihrer ersten Maßnahmen war der Abbruch der Verhandlungen über einen streng konditionierten IWF-Kredit. Die wichtigste Bedingung staatlicher und privater Gläubiger-Banken für die Refinanzierung der fälligen Außenschuld wurde so in den Wind geschlagen. Folglich wurde auch die Möglichkeit, einen Mehrjahresvertrag über die Restrukturierung der Außenschuld abzuschließen, praktisch annuliert. 
Im Rahmen des wachsenden Widerstands in ganz Lateinamerika gegenüber den Forderungen der ausländischen Gläubiger erhielt diese Haltung der brasilianischen Regierung die Bedeutung, die Wirtschaftspolitik der verschuldeten Länder nicht mehr unter die Oberaufsicht des IWF unterzuordnen. Bis 1985 wurde dieser Forderung nur von kleineren und mittleren Schuldnern Widerstand entgegengesetzt, während die drei größten Schuldner - Brasilien, Mexico und Argentinien - sich ihr unterwarfen. So wurde der Schein gewahrt, daß eine sogenannte konventionelle Behandlung der Auslandsschulden gewährleistet sei. Die Positionsänderung der brasilianischen Regierung - obwohl mit höchster Diskretion vorgenommen - signalisierte die Rebellion der demokratischen Regierungen (wie auch der von Venezuela, Peru und von der Dominikanischen Republik) gegen die Forderungen des Auslands nach wirtschaftspolitischen Regulierungsmechanismen, die den Interessen der ausländischen Gläubiger dienten, aber mit schwersten Kosten für die Arbeiterklassen und das nationale Kapital verbunden waren. In der weltweiten Auseinandersetzung um die Verschuldungskrise der Dritten Welt stärkte die von Brasilien eingenommene Position die Kräfte, die sich der erwähnten »konventionellen Behandlung « in den Schuldner-, aber auch in den Gläubigerländern widersetzten.

Ende 1985 waren das Wirtschaftskabinett und selbst Sarney davon überzeugt, daß der einzige Weg der Inflationsbekämpfung eine Währungsreform auf der Grundlage des »neostrukturalistischen « Vorschlags sei. Es wurde unter höchster Geheimhaltung eine Arbeitsgruppe gebildet, die Maßnahmen vorbereiten sollte, um den »heterodoxen Schock « ohne Vorankündigung in die Wirklichkeit umzusetzen. Der »Cruzeiro《 sollte durch den »Cruzado« ersetzt werden, jeder Cruzado 1000 Cruzeiros wert sein. Die Löhne sollten mit Hilfe einer Tabelle in Cruzados umgerechnet werden, die jedem Arbeiter einen Lohn in der Höhe des durchschnittlichen Realeinkommens des Vormonats zubilligte. Eine andere Tabelle sollte für die Umrechnung der Cruzeiro-Schulden in Cruzado-Schulden benutzt werden, deren Wert um die »zu erwartende « Inflation (ein täglicher Abwertungssatz von $0,5 \%$ ) bereinigt werden sollte. Dies wurde damit begründet, daß in jeder Schuldensumme schon eine vorweggenommene monetäre Korrektion enthalten sei, die der zu erwartenden Entwertung des Geldes bis zur Fälligkeit entspricht. Wenn die Schulden also »gleich« konvertiert würden $(1000 \mathrm{Cr} .=1 \mathrm{Cz}$.), würden sich die Gläubiger zum Schaden der Schuldner bereichern. Es muß noch erwähnt werden, daß die Konversionstabellen der Löhne wie auch der Schulden davon ausgingen, daß ab dem »choque heterodoxo« die Inflation gleich Null sei.

Etwas mehr als eine Woche vor dem Schocktag stellte die Arbeitsgruppe fest, daß die Preiskontrollorgane keine Möglichkeit hatten, die Umrechnung der Preise in Cruzados auf der Basis der durchschnittlichen Realpreise des letzten Halbjahres durchzuführen. Nun entschloß man sich, in Ersetzung dieser Maßnahme die Preise auf dem Niveau des Schocktages einzufrieren. Dieser Vorschlag wurde enthusiastisch vom Präsidenten aufgenommen, der intuitiv spürte, daß ein generelles Ein- 
frieren der Preise den tiefgehenden Bedürfnissen der Massen entgegenkam und so deren breite Unterstützung des Plans garantierte. Trotzdem blieben die Autoren des Planes besorgt hinsichtlich der Reaktion der Lohnabhängigen. In Israel und Argentinien sind die »heterodoxen Schockmaßnahmen« von der Gewerkschaftsbewegung stark angefeindet worden. Die Festlegung der Löhne »nach dem Durchschnitt« (der letzten drei Monate) verursachte unverzüglich Nominallohnsenkungen bei den Arbeitern, deren Löhne in den letzten zwei Monaten an die Inflation angeglichen worden waren - was trotz eingefrorener Preise eine augenblickliche Reallohnsenkung zur Folge hatte. Das gleiche würden den Arbeitern geschehen, deren Lohnangleichung mit dem Beginn des Planes zusammenfiele. Denn ihre Löhne würden viel weniger steigen als die Inflationsrate seit der letzten bezogenen Angleichung. In jedem Fall war der Durchschnittslohn niedriger als der letzte Spitzenlohn, der von den Arbeitern oder zumindest ihren Gewerkschaftsvertretern als die gerechte Lohnhöhe angesehen wurde, die ihnen aber inzwischen von der Inflation »geklaut« worden war.

Um »die Pille zu versüßen «, beschloß die Regierung, im Zuge von Verhandlungen zwischen den Ökonomen, die den Plano Cruzado formuliert hatten, und dem Arbeitsministerium in letzter Minute, den Durchschnittloshn um eine Zugabe von $8 \%$ und den Mindestlohn um einen Satz von $16 \%$ zu erhöhen. Außerdem wurden in den »Plano Cruzado« noch zwei alte Forderungen der Arbeiterbewegung aufgenommen. Die Arbeitslosenversicherung und die gleitetende Lohnskala. Beide Maßnahmen waren so angelegt, daß sie nicht viel Wirkung zeitigen konnten: Die Arbeitslosenversicherung beschränkte sich auf die Gewährung eines monatlichen Mindestlohnes nur für diejenigen Arbeiter, die vorher mindestens drei Jahre in die Sozialversicherung eingezahlt hatten; die automatische Lohnangleichung sollte einsetzen, wenn die Lebenshaltungskosten um mehr als $20 \%$ stiegen. Die Erwartung war natürlich, daß dies mit dem »Plano Cruzado« gerade nicht eintreten würde.

Am 28. Februar 1986 kündigte der Präsident, begleitet von den Finanz-, Planungsund Arbeitsministern, im Fernsehen den »Plano Cruzado« an. In seiner Rede verkündete Sarney, daß von diesem Moment an die Preissteigerungen passé seien, und er appellierte an die Zuschauer, ihm bei der Kontrolle der Einfrierung zu helfen, ja, er ernannte alle Bürger symbolisch zu »Preisaufsehern des Präsidenten«. An jenem und an der folgenden Tagen beherrschten die Währungsreform und die neuen Mechanismen der Wirtschaftsregulierung die Medien. Sie waren Gegenstand leidenschaftlicher Diskussionen in allen Ecken des Landes.

Die Aufnahme des Plans war insgesamt positiv. Für seine begeistertsten Verfechter bedeutete der Plano Cruzado die endgültige Auslöschung der brasilianischen Inflation, zusammen mit der Handels- und Finanzspekulation, was die Glaubwürdigkeit, Ehrlichkeit und Aufwertung der Arbeit mit sich bringen würde ... Der Cruzado-Plan war für viele nichts weniger als die nationale Errettung, die langersehnte Einlösung der Versprechen, die mit dem Sturz des Militärregimes verbunden und durch den unerwarteten Tod Tancredo Neves enttäuscht worden waren. Für die Skeptiker war 
der Plano Cruzado eine neue und geistreiche Form der Inflationsbekämpfung mit dem großen Vorteil gegenüber den orthodoxen Rezepten, daß die Wirtschaft nicht in eine Rezession gestürzt wird. Die große Mehrheit der Kommentatoren mit Zugang zu den Massenmedien bewegte sich zwischen diesen Positionen, wobei nachgerade bombastische Formulierungen gebraucht wurden, die selbst die Intellektuellen an der Macht ansteckten. Diese gingen ebenfalls bei ihren häufigen TV-Auftritten dazu über, den Plano Cruzado als die fast magische Lösung aller ökonomischen Probleme darzustellen.

Natürlich gab es auch kritische Stimmen, hauptsähclich aus der Linken und aus der Gewerkschaftsbewegung. Der PT und der PDT verurteilten den Plano Cruzado hauptsächlich wegen der »Knebelung « der Löhne bzw. wegen ihrer nominalen Senkung, während doch die Preise auf ihrem »Spitzenniveau« eingefroren würden. Die Gegenargumentation der Verteidiger des Planes lief darauf hinaus, daß ein Teil der Preise sich gerade vor der Erhöhung befand - einige waren bereits beschlossen - und daß diese Preissteigerungen von der Regierung zurïckgenommen wurden, so daß auf diese Weise eventuelle Lohneinbußen kompensiert werden konnten. Die härtesten Kritiken kam von den beiden Gewerkschaftsdachverbänden DGT, (PMDB, PCB und PC do B nahestehend) und der CUT (dem PT nahestehend); letztere sprach sogar von einem Generalstreik gegen den Plano Cruzado. Aber die Gewerkschaftsbasis teilte die kritische Haltung ihrer Funktionäre nicht und tauschte guten Mutes geringere Nominallöhne gegen eingefrorene Preise. In den ersten Monaten der Laufzeit des Plano Cruzado schwächte sich die Mobilisierungsfähigkeit der Gewerkschaften so stark ab, daß nicht wenige Gewerkschaftsführer überzeugt davon waren, der Hauptzweck des »choque heterodoxo« sei die Schwächung der Arbeiterbewegung gewesen.

Am beeindrucktendsten war die Reaktion der Massen auf den Plano Cruzado. Seit dem 28.2.86 gingen viele Verbraucher in die Supermärkte und Warenhäuser, um die Respektierung der Preistabellen einzufordern. In vielen Supermärkten wurden Angestellte bei der fleißigen Neuauspreisung von vielen Artikeln überrascht. Die größte Supermarktkette des Landes beschloß, als sie am Vorabend der Preiseinfrierung davon erfuhr, die Preise generell um ein Drittel anzuheben. Andere Ketten versuchten das gleiche. Die Verbraucher - in ihrer Rolle als »Sarney's Preisaufseher «stürmten massenhaft die Supermärkte, von denen viele daraufhin geschlossen wurden. Die Polizei ging dazu über, die Verkaufsleiter festzunehmen, hauptsächlich um sie dem Massenzorn zu entziehen. Trotzdem wurden Fälle von Plünderungen aus verschiedenen Städten gemeldet. Einige Tage später veröffentlichten die Zeitungen »offizielle« Preistabellen von hunderten von gebräuchlichen Konsumgütern. Extraauflagen in Millionenhöhe waren schnell ausverkauft. Käufer organisierten sich spontan, um die Läden aufzusuchen und die Preiseinhaltung zu sichern. Hausfrauenvereinigungen stürzten sich eifrig auf diese Aufgabe, zeigten die Gesetzesübertreter an und einige Male setzten sie direkte Festnahmen und Schließungen des Ladens durch. Die TV-Nachrichten verbreiteten diese Szenen täglich und stachelten 
den patriotischen Ehrgeiz der »Preisaufseher des Präsidenten« an. Viele Ladenbesitzer erklärten sich zum »Opfer regelrechter Pogrome«, da sie von der öffentlichen Meinung als »Saboteure« des Plano Cruzado dargestellt würden.

Einige Monate später wurden die Linksintellektuellen an der Macht auf einer Sitzung zur Evaluierung des Plano Cruzado von Sarney mit folgenden Worten begrüßt: »... Sie können sich nicht vorstellen, was Sie für das Land getan haben. Das war keine einfache ökonomische Wende. Sie wollen es vielleicht nicht wahrhaben, aber Sie haben mit einer sozialen Revolution begonnen.«Und der Finanzminister Dilson Funaro steigerte dieses Lob noch: »Sie haben Geschichte gemacht! «. Solche Lobessprüche erschienen zu dieser Zeit nicht übertrieben. Nur in seltenen Fällen sind Intellektuelle an die Macht gekommen, um ein eindeutig akademisches Programm in die Praxis umzusetzen, das zu keinem Zeitpunkt von den maßgeblichen politischen Kräften des Landes ernsthaft diskutiert worden wäre. Strenggenommen verstanden weder der Präsident noch der Finanzminister, und noch weniger die laienhaften politischen Führer inner- und außerhalb der Regierung die ökonomische Theorie, auf der der Plano Cruzado beruhte. (»... Die Regierung lancierte ein Programm, bei dem sie selbst nicht verstanden hatte, um was es sich handelte, « erklärte P. Arida). Wichtig war nur, daß er funktionierte oder zu funktionieren schien. Durch ein überraschend verkündetes Dekret wurden neue Regeln der Preis-, Lohn-, Zins-, Wechselkurs-, Mietfestsetzungen eingeführt und die Inflation verschwand. Während des ersten Monats sank das Preisniveau um etwas weniger als ein Prozent. In den Folgemonaten waren die Steigerungen minimal im Vergleich zur zweistelligen Inflationsrate, die zuvor vorherrschte. Die Zufriedenheit der Bevölkerung war enorm, und Meinungsumfragen ergaben, daß Sarney mit $90 \%$ der Zustimmung der Bevölkerung rechnen konnte.

Selbst die Intellektuellen an der Macht hatten keine richtige Vorstellung davon, was sie in Gang gesetzt hatten. Mitten in der allgemeinen Begeisterung konnten sie nicht weniger begeistert sein. Aber es muß auch gesagt werden, daß sie anfingen daran zu zweifeln, ob der erreichte Erfolg ein dauerhafter sein könne. Während des erwähnten Treffens zur Einschätzung des Plans, in der sie die überschwenglichen Glückwünsche des Präsidenten und ihres Finanzministers erhielten, formulierten sie bereits ihre Befürchtungen, daß die Inflation möglicherweise zurückkommen könne. Obwohl die Gründe falsch waren (wie wir noch sehen werden) - ihre Intuition war richtig. Aber dieses Mal wurde nicht auf sie gehört, denn die Politiker waren nicht gewillt, auf ihren Popularitätsgenuß zu verzichten. Später verstanden sich Politiker und Intellektuelle immer weniger, im gleichen Maße wie die ökonomischen Daten sich verschlechterten. In spektakulären Abfolgen von Mißverständnissen, Zerwürfnissen und Zusammenstößen kam das bittere Ende. »Sic transit gloria mundi.« 


\section{Leben, Passion und Tod des Plano Cruzado}

Die Einfrierung der Preise auf dem Niveau vom 28.2.86 war von Anfang an unpraktikabel. Im März beginnt in Brasilien der Übergang vom Sommer in den Herbst, die Kleidergeschäfte verkaufen ihre Restbestände im Sommerschlußverkauf und stellen die neue Modekollektion vor. Der Wechsel der Modelle macht einen Preisvergleich unmöglich. Selbst im März, im ersten Monat der Preiseinfrierung, waren die Bekleidungspreise recht hoch, aber kompensiert durch die niedrigen Lebensmittelpreise, denn in dieser Jahreszeit kommen die Ernten auf den Markt. Die Preise für bestimmte Waren, wie Kleidung, Schuhe, Möbel, Spielzeug etc. waren überdies nicht eingefroren. Das gleiche war der Fall bei Dienstleistungen von Kleinunternehmern wie Friseursalons, bei Leistungen der Krankenhäuser und Zahnkliniken, von Reparaturwerkstätten, Reinigungen etc.

Das Einfrieren hatte eigentlich nur bei den geschützten Produkten Wirkung, und hier tauchten auch zugleich Schwierigkeiten auf. Wegen der Intensität der vorangegangenen Inflation waren die Preise von vielen Produkten auf einem Niveau nahe oder sogar unter dem Gestehungspreis eingefroren. Weil aber nur die Preise der Endprodukte festgelegt waren, schlugen die Ökonomen in der Regierung vor, daß die Branchen mit ungenügenden Gewinnen oder mit Einbußen von ihren Lieferanten einen Preisabschlag (Skonto) verlangen könnten. Diese Forderung war sinnvoll, denn Rohstoffe, Komponenten etc. werden mit Lieferfristen verkauft und schließen in ihrem Preis einen Inflationsvorschuß ein, der durch den Plano Cruzado nun wegfiel. Über die Höhe des impliziten Vorschusses, also um die Größe des Skontos, entwikkelten sich vielfältige Konflikte, die sogar viele Geschäftstransaktionen in vielen Sektoren zum Erliegen brachten. Im März trat sogar eine Mini-Rezession ein, weil eine Einigung über die Preise zwischen den einzelnen Wirtschaftssektoren, zwischen Großindustrie und Kleinhandel etc. nicht zustande gekommen war. Die Regierung weigerte sich, als Schiedsrichter in diese Konflikte einzugreifen. Die Ökonomen der Regierungsgruppe waren überlastet mit unzähligen Detailfragen, wie mit Regeln für die Berechnung der Mieten für Wohnungen und Läden, Schulgelder, Versicherungsprämien, Steuerbescheide etc. in der neuen Währung Cruzado. Es erschien ihnen unmöglich, die unzähligen Konflikte zu schlichten, von denen jeder einzelne von besonderen Begleitumständen und sektoriell gegensätzlichen Interessen geprägt war. Aus Bequemlichkeit, Hoffnungslosigkeit oder Überzeugung vertrauten sie dem Marktmechanismus die Lösung der Konflikte zwischen den Wirtschaftssektoren an, die durch den »choque heterodoxo « hervorgerufen worden waren. Im theoretischen Modell sollten die Preise ein Gleichgewicht stabilisieren, wenn die Dynamik der inertialen Inflation erst einmal beseitigt war. Praktisch war der Markt Schauplatz von Verteilungskämpfen, die über seine Grenzen hinausreichten und die institutionalisierten Spielregeln in Frage stellten. Letztendlich lösten sich die Konflikte dadurch, daß die eingefrorenen Preise, die ihre Ursache gewesen waren, wieder heraufgesetzt wurden. 
Eine andere unerwartete Begleiterscheinung - jedenfalls aus der Sicht der neostrukturalistischen Theorie - war der starke Konsumboom seit Inkrafttreten des Plano Cruzado. Die Bevölkerung frequentierte in höchstem Maße die Läden, Restaurants, Kinos, Flughäfen und Hotels, es kam zu Verkehrsstockungen und ständiger Überbuchung der Verkehrsmittel, der Ausflugslokale und sogar der Krankenhäuser. Die Konsumsteigerung wurde mit den höheren Lohneinkommen infolge der gleichzeitigen Erhöhung aller Löhne auf den Durchschnittslohnsatz, der Zugaben von $16 \%$ auf den Mindestlohn und der $8 \%$ auf alle Löhne erklärt. In Wirklichkeit war die Plünderung der Sparguthaben viel entscheidender als die Lohnerhöhungen (die in Wirklichkeit sehr gering waren). Die Sparkonten wurden der monatlichen Inflation angeglichen und mit $0,5 \%$ pro Monat verzinst. Mit dem Rückgang der Inflation auf fast Null erhielt man nur noch 0,5\% monatliche Zinsen auf die Sparguthaben. So meinten die (in der Geldillusion befangenen) Leute, vor dem Plano Cruzado hätten sie monatlich $15 \%$ Zinsen und nach dem Plano Cruzado nur $0,5-1 \%$ Zinsen verdient. Offensichtlich zogen die Verbraucher von den Zinseinnahmen nicht die durchschnittliche Steigerung der Lebenshaltungskosten ab, was zwar für den Ökonomen, aber nicht für den normalen Bürger selbstverständlich ist. Vor dem Plano Cruzado lebten nicht wenige Sparer ganz oder zum Teil von der Geldwertkorrektur, indem sie die Zinsen abhoben und von ihnen wie von einem Einkommen lebten, obwohl sie de facto ihr Kapital auffraßen. Als dieses fiktive Einkommen mit dem Ende der Inflation verschwand, plünderten viele Sparer ihre Guthaben, um Gebrauchsgüter und Dienstleistungen zu erstehen, die durch das Einfrieren aller Preise überraschend billig geworden waren.

Der Druck der Konsumsteigerung führte zu Preissteigerungen bei den nicht fixierten Preisen und zunächst zu Steigerungen des Angebots bei den Waren mit de facto eingefrorenen Preisen. Da letztere den Großteil des Warenkorbs ausmachten, war der Anfangseffekt auf die Lebenshaltungskosten klein. Aber nach und nach begannen Waren, deren Preise auf niedrigem Niveau eingefroren worden waren, aus den Supermärkten und Warenhäusern zu verschwinden, und konnten dann nur noch in kleinen Läden, die nur schwer kontrollierbar waren, zu erhöhten Preisen erstanden werden. Die so verursachten Versorgungsengpässe entzogen den »Preisaufsehern des Präsidenten« die Grundlage: Sie erhielten keinerlei Regierungsunterstützung, um die Hinterziehung der Waren und die Schwarzmarktpraktiken zu verhindern. Es gibt keine plausible Erklärung für die Untätigkeit der Regierung bei der Überwachung der eingefrorenen Preise. Sofort nach dem Dekret gab es Hinweise der Bundesregierung auf die Einrichtung eines großen Apparats von Preiskontrolleuren und Versorgungsbehörden. Aber die Absichten wurden nie in die Wirklichkeit umgesetzt. In wenigen Wochen war die spontane Kontrolle völlig demoralisiert und hörte dann auch auf. Die Untätigkeit der Regierung läßt sich möglicherweise durch die Tatsache erklären, daß einige der Ökonomen, die den Plano Cruzado mit verfaßt und hatten anlaufen lassen, das Einfrieren der Preise sowieso nur als kleineres Übel ansahen. Z.B. erklärte Pérsio Arida später: »André Lara Rezende und ich mochten das 
Einfrieren der Preise nicht (...). Als man einer kleinen Zeitspanne der Einfrierung zustimmte - allerhöchstens 3 Monate - dachten wir, eine kurze Zeit würde keinen groBen Schaden anrichten. Teufel auch, die 3 Monate dauerten elf und daher kam, was kommen mußte. Wir haben letztendlich dem Einfrieren der Preise zugestimmt. « Für eine dreimonatige Preiseinfrierung war es allerdings nicht notwendig, einen umfangreichen Kontrollapparat, der wenigstens minimale Effizienz zeitigen würde, $\mathrm{zu}$ installieren und zu mobilisieren. Auch war gar keine Zeit dazu. Aber gleich im April entschied der Präsident, daß die Preisfestschreibung nicht aufgehoben würde, wenn nicht der große Druck auf die Preise nachlassen würde. Diese Entscheidung wurde in der Öffentlichkeit von den Ministern Funaro und Sayad wie von den anderen Intellektuellen an der Macht verteidigt. Aber die Ökonomen, die ohne eigenes Wissen »eine soziale Revolution eingeleitet hatten «, hegten weder enthusiastische Gefühle gegenüber den Millionen Hausfrauen, die den Plano Cruzado unterstützten, noch dachten sie daran, sich auf diese zur Konsolidierung der Erfolge zu stiutzen. Ihre gesamte Strategie war strikt makroökonomisch, auf der Manipulation der Marktmechanismen basierend. Nach ihrer Ansicht war die Wirtschaft überheizt und der Nachfrageüberhang würde die Inflation zurückbringen. Also ging es nicht darum, die eingefrorenen Preise durch staatliche Maßnahmen im Zusammenspiel mit der spontanen Massenuntersützung zu kontrollieren, sondern die Nachfrage durch Zinserhöhungen, öffentlichen Ausgabenstop und Steuererhöhungen abzukühlen. Ab diesem Moment waren alle Maßnahmen der Linksintellektuellen an der Macht auf dieses Ziel gerichtet und sie versuchten, die politische Führung von der Notwendigkeit ihrer Durchführung zu überzeugen.

Im August 1986 begann die bis dahin unterdrückte Inflation offen auszubrechen. Die Bezahlung eines »agio« oberhalb des in den Tabellen ausgewiesenen Preisniveaus setzte sich durch, auch auf die Rohstoffe. Die Großindustrie, die verpflichtet war, sich an die Preisobergrenzen zu halten, war in der Lage gewesen, ihre Gewinne wegen der Produktionserhöhungen und der vorgenommenen Einsparungsmaßnahmen auszudehnen. Aber viele andere Industrien, die Halbfertigwaren herstellten, konnten bei dieser Expansion nicht mitziehen. Die Verteuerung der inputs begann die Profitmargen der Konsumgüterindustrie zusammenzudrücken. Nach und nach verengte sich der Bereich, in dem die Preise noch eingefroren waren. Es häuften sich die Fälle, in denen die Produktmarkte gewechselt wurde, um das Einfrieren zu vermeiden (die Preise waren nach Produktmarken tabelliert) und die »agios « wurden jedesmal höher. Die offizielle Inflationsrate verlief 1986 wie folgt: August 1,68\%, Sept. 1,72\%, Okt. $1,90 \%$, Nov. $3,29 \%$, Dez. $7,27 \%$.

Im November waren Wahlen, bei denen der PMDB der große Gewinner war. Weniger als eine Woche nach den Wahlen verabschiedete die Regierung ein neues Paket restriktiver Maßnahmen, das »Cruzado II « genannt wurde. Das neue Paket folgte der gleichen Logik wie das vorangegangene, ersetzte nur die Zwangsanleihen durch die Erhöhung der indirekten Steuern, der Tarife öffentlicher Dienstleistungen und der Preise von Produkten der Staatsbetriebe. Die Reduzierung der Nachfrage sollte 
durch die Verteuerung der Lebenshaltungskosten erreicht werden, ohne daß die Löhne erhöht würden. Dies machte eine Änderung des offiziellen Inflationsindexes erforderlich: die Produkte wurden einfach aus dem Warenkorb herausgenommen, deren Preise durch den »Cruzado II « erhöht worden waren.

Die Reaktion der öffentlichen Meinung war, wie zu erwarten war, extrem kritisch. Die gleichen Massenmedien, die im März von der Zauberkraft des Plano Cruzado begeistert waren, bezichtigten im November die Regierung des Verrats an den Wählern, weil sie ihr unpopuläres Paket direkt nach den Wahlen herausgelassen hätte. Praktisch niemand, außer den Regierungssprechern, verteidigte oder rechtfertigte den $\gg$ Cruzado II $\ll$. Die Linksintellektuellen, die angesichts des Inkrafttretens des Cruzadoplanes die fast einhellige Unterstïtzung der Bevölkerung geerntet hatten, waren neun Monate später völlig isoliert und sahen sich einem Meer von Kritiken aus allen Parteien (ihrer eigenen eingeschlossen) und von allen sozialen Gruppen gegenüber.

Die Rechte tadelte die Regierung wegen ihrer zu laschen Ausgabenbeschränkungen, wegen der zu geringen Steuer- und Preiserhöhungen der staatlichen Dienstleistungen zur Deckung des Defizits. Aus diesem Blickwinkel wurde der »Cruzado II « als gigantische Expropriation des Privatgewinnes zur Fütterung der unersättlichen Staatsmaschinerie gesehen. Die Linke kritisierte den »Cruzado II « wegen seiner inflationistischen Wirkung und der weiteren Reallohnsekung infolge der Preissteigerungen. Im Grunde stimmte die Rechte bei der makroökonomischen Diagnose mit den »Vätern des Cruzado « überein, verlangte aber von der Regierung, daß sie die Preiseinfrierung aufhöbe und durch Reduzierung der öffentlichen Ausgaben die Inflation eindämme. Die Linke stimmte mit der Sicht der Regierung überein, als diese die Preiseinfrierung als Inflationsbarriere proklamiert hatte. Deshalb beschuldigte sie die Regierung der Kapitulation vor dem Großkapital, weil sie die Preise jeglicher Kontrolle entgleiten ließ und weil sie in brutaler Form die Preise der Güter und Leistungen erhöhte, die ihrer direkten Verantwortung unterlagen. Die durch die Modifizierung des offiziellen Infaltionsindex IPC hervorgerufene Empörung war so groß, daß die Regierung gezwungen war, zurückzustecken und den Inflationsindex so zu lassen wie er war.

Der Cruzado II « war ein bemerkenswertes Beispiel für politische Unfähigkeit, erwachsen aus einem totalen Unverständnis über das, was die politisch Verantwortlichen und die ökonomischen Berater beabsichtigten. Alles weist darauf hin, daß Sarney und Funaro nie die orthodoxe makroökonomische Logik verstanden hatten, die der Strategie der Ökonomengruppe zugrunde lag (die zu diesem Zeitpunkt schon vollends uiber taktische Divergenzen zerstritten war, was hier aber nicht zur Debatte steht). Der »Cruzado II « war der Gnadenschuß, der die Reste des Plano Cruzado beseitigte. Wenn die Regierung schamlos die Preise der Produkte von Staatsbetrieben und die öffentlichen Tarife erhöhte, gab es auch keine moralische Autorität mehr, die die Preisobergrenzen stützen konnte. Diejenigen, die versteckte »agios« praktiziert hatten, gingen dazu über, sie offen aufzuschlagen. Deshalb sprang die monatliche 
Inflationsrate im Dezember auf 7,27\% und erreichte im Januar 1987 16,82\%. In weniger als einem Jahr war die Inflation triumphierend zurückgekehrt und übertraf (1987 auf das Jahr umgerechnet mit über 451\% - Anm. der Redaktion) das Niveau, das sie vor dem Plano Cruzado erreicht hatte.

\section{Epilog}

Formal wurde die Preiseinfrierung erst im Februar 1987 aufgehoben, als selbst die teilweisen Preiskontrollen aufgegeben wurden, die es vor dem Plano Cruzado gegeben hatte. Im gleichen Monat begann der größte Teil der für den ersten »heterodoxen Schock « Verantwortlichen die Regierung zu verlassen. Es gingen die meisten, die in der Zentralbankdirektion gearbeitet hatten, danach der Planungsminister und im April auch der Finanzminister. Die Zeit der Machterfahrung der Linksintellektuellen schien abgeschlossen. Aber tatsächlich trat sie nur in eine zweite Phase, die hier nur kurz angeschnitten werden soll.

Der Rücktritt von Funaro als Finanzminister leitete einen offenen Konflikt zwischen Sarney und Ulysses Guimaraes ein, aus dem die Nominierung von Luiz Carlos Bresser Pereira, einem anderen Linksintellektuellen (der im Dezember 1987 seinerseits wieder zurücktrat - Anm. d. Ü) hervorging. Dieser Ökonom hatte bei der Vargas-Stiftung (FGV) in Sao Paulo selbst eine Theorie der »inertialen Inflation « entwickelt, die in vielen Punkten der der Professoren von der PUC-Rio entsprach. Deshalb unterstützte Bresser den Plano Cruzado uneingeschränkt und reklamierte praktisch eine Mitautorenschaft an diesem theoretischen Ansatz. Als Finanzminister bildete er eine Beratergruppe aus Wirtschaftswissenschaftlern des PMDB, wobei er versuchte, auch Mitglieder der vorherigen Gruppe zu integrieren. Man kann sagen, daß der Wechsel von Sayad und Funaro zu Bresser weit davon entfernt war, die wirtschaftspolitische Linie der Regierung zu unterbrechen, im Gegenteil: sie wurde mit der teilweisen Umbesetzung der Führungspositionen bestärkt.

Zwischenzeitlich besorgte die Rückkehr einer heftigen Inflation das schmutzige Geschäft, das die aufeinanderfolgenden wirtschaftspolitischen Pakete nicht geschafft hatten. Die immens hohen Lebenshaltungskosten reduzierten die Kaufkraft der Lohnabhängigen und führten zu einer drastischen Nachfragesenkung. Die gleitende Lohnskala begann bei den Löhnen zu wirken, aber immer mit einem beachtlichen Rückstand gegenüber den Preissteigerungen. Die Umsätze im Handel waren rückläufig und eine sofortige Rezession wurde nur dadurch verhindert, daß Industrie und Handel die Lager, die in der Krisenzeit des Plano Cruzado geleert worden waren, wieder auffüllten. Ab Mai/Juni erreichte der Rückgang auch die Großhändler und die Industrie reagierte auf die fehlenden Bestellungen mit Produktionseinschränkungen und Entlassungen.

Bresser beendete den zweiten »choque heterodoxo« im Juni 1987 und versuchte mit allen Mitteln, die Fehler zu vermeiden, die er und seine Kollegen der »Heterodoxie« 
als fatal am Plano Cruzado betrachteten. Diesmal gab es keine Gnade für die Lohnabhängigen. Die gleitende Lohnskala wurde abgeschafft, die Löhne nun monatlich an die Inflation des letzten Trimesters angepaßt. Eine letzte Anpassung um 20\%, die den Lohnabhängigen im Juli zugestanden hätte, wurde gestrichen. Die Reallohneinbußen, die aus dem inflationären Schub des ersten Halbjahres 1987 resultierten, wurden somit nicht kompensiert und also verewigt. So wurde eine explizite Politik der »Lohndrosselung « exekutiert, damit es diesmal garantiert keine Nachfrageexzesse gäbe. Die Preise wurden nur für drei Monate eingefroren, um danach langsam wieder liberalisiert zu werden. Der sogenannte »Breser-Plan« schloß auch noch eine Währungsabwertung und eine starke »korrektive Inflation《 ein, mit Preiserhöhungen im öffentlichen Sektor und bei Getreideprodukten, deren Subventionen gekürzt wurden. So wurde die heterodoxe Maßnahme der Einfrierung durch einen Maßnahmenkatalog vervollständigt, der offen und eindeutig die Absicht verfolgte, die Löhne und damit die Nachfrage sowie das Ausgabendefizit zu senken. Diesmal wurden die neostrukturalistischen Rezepte unter fast idealen Bedingungen in die Praxis umgesetzt ... aber trotzdem scheiterten sie.

Da der Bresser-Plan keinerlei Begeisterung in der Bevölkerung hervorrief, hatte auch die Einfrierung der Preise viel weniger Erfolg. Die Inflation fiel praktisch nie unter 5\% monatlich, und als die Einfrierung ab September offiziell beendet war, stieg sie wieder und erreichte 9,18\% im Oktober und 12,84\% im November. Der zweite Schock, der in Realität mehr orthodox denn heterodox war, erwies sich als viel unwirksamer als der erste. 1987 folgte der »korrigierten « Neuauflage des »heterodoxen Schocks« die »Stagflation«.

\section{Schlußfolgerungen}

Im Rahmen der kürzlichen und noch nicht abgeschlossenen brasilianischen Redemokratisierung war nur ein Teil der Linksintellektuellen an die Macht berufen worden: die Ökonomen, die zur alten Opposition gegen das Militärregime gehörten. Diese Tatsache charakterisiert die Ambivalenz des Übergangsprozesses, in dem Fortschritte im sozialen Bereich infolge der Verschärfung der Verteilungskonflikte, die sich in sukzessiven inflationären Explosionen äußern, tatsächlich blockiert sind. Brasilien wurde 1982 unter im Vergleich mit anderen Ländern relativ privilegierten Bedingungen von der Schuldenkrise erfaßt: es besitzt einen großen, ziemlich vereinheitlichten Binnenmarkt, die nationale Industrie ist genïgend diversifiziert und verfügt über ein elastisches, billiges Arbeitskräftepotential und gleichzeitig über fähige technische und administrative Kader, die sich schnell an den technischen Fortschritt anpassen können. Deshalb konnte Brasilien in ausreichendem Maße Importe substituieren und Exporte diversifizieren, also eine positive Handelsbilanz erwirtschaften, mit deren Saldo die Zinsen auf die enorme Außenschuld 1984 ganz 
bezahlt werden konnten. Gleichzeitig war es der Wirtschaft möglich, die Rezession zu überwinden und scheinbar einen neuen Wachstumszyklus auf der Basis der Ausweitung des Binnenmarktes zu beginnen.

Aber die Wachstumspolitik unter den Bedingungen des Übergangs zur Demokratie verlangte einen ökonomischen Strukturwandel, der gleichzeitig die Erhöhung der Reallöhne, die von drei Rezessionsjahren ausgehöhlt waren, die Ausweitung des Staatsanteils insbesondere für Sozialausgaben (für Bildung, Gesundheit, Wohnungsbau etc.) beinhaltete und die Investitionen in der Grundstoffindustrie und bei den Infratstrukturmaßnahmen steigerte. Diese Umstrukturierung kann natürlich nur auf Kosten irgend eines anderen Sektors gehen, jedenfalls zeitweise. Dieser andere Sektor hätte derjenige der ausländischen Gläubiger sein können, die vor dem Moratorium 4 - 5\% des BIP absorbierten; und/oder das hauptsächlich von der Staatsschuld profitierende Finanzkapital, das 1985 11,5\% des Volkseinkommens in Form von Zinsen auf Schuldverschreibungen an sich ziehen konnte.

Die brasilianische Inflation ist also Verteilungskonflikten geschuldet, in die Auslandsgläubiger, Staat, nationale produktive Bourgeoisie, Bankkapital, Kleinbürgertum und Lohnabhängige einbezogen sind. Diese vielfältigen, gleichzeitigen, miteinander vernetzten Konflikte führen nicht zur notwendigen Umstrukturierung, wenn sie auf die Ökonomie beschränkt bleiben. Die Inflation verhindert ihre Lösung. Die Inflationsdynamik verändert die Einkommensverteilung ständig und erlaubt nicht, daß irgendein Sektor sein Stück vom Kuchen beständig verkleinert oder vergrößert. Damit die Verteilungskonflikte in einer Art und Weise ablaufen, die das Wirtschaftswachstum stützen könnte, und die Forderungen der Mehrheit, die nach demokratischen Regeln Vorrang haben sollten, berücksichtigen, ist es notwendig, diese Konflikte auf die Ebene des politischen Systems zu heben. Nur dort kann sich eine mögliche Koalition von mehrheitsfähigen Interessen bilden, die die Inflation überflüssig machen und durch wirtschaftspolitische Regulierungsmechanismen ersetzen könnte.

Natürlich impliziert dies die Bildung neuer Institutionen zur Verhandlung von Konflikten und zur außerökonomischen Intervention bei der Schaffung, Aneignung und Nutzung des gesellschaftlichen Überschusses. Institutionen dieser Art, wie sie schon in einigen entwickelten europäischen Demokratien existieren, bilden sich nicht über Nacht. Sie sind Ergebnis eines langen Prozesses von Versuchen und Niederlagen, in denen die Klassen den unverzichtbaren Lernprozeß im konfliktreichen Zusammenleben in einer heutigen Demokratie durchmachen. Die heterodoxen Wirtschaftswissenschaftler jedoch gingen von der Annahme aus, daß die Inflation die Verteilungskonflikte ausgeglichen habe und deshalb überflüssig geworden wäre und durch eine von oben verordnete Neuregelung der Preisbildungsmechanismen ausgelöscht werden könnte.

Als die ersten Wirkungen des Schocks vorbei waren, war es nicht das erwartete Gleichgewicht, das zum Vorschein kam, sondern eine neue Inflationswelle. Die Linksintellektuellen an der Macht zogen ihre anfängliche Annahme zurück und 
machten sich daran, das Gleichgewicht durch konventionelle makroökonomische Kontrolle der Nachfrage herzustellen. Hier verbrauchte sich ihre Heterodoxie und ihre fiskal- und geldpolitischen Vorschläge erwiesen sich als politisch nicht umsetzbar. Beim Versuch, die »inertiale« Inflation auszuschalten merkten sie, daß diese wenig »Intertiales« hatte. Sie wurden folglich dazu gezwungen, die Einkommen umzuverteilen, um die Nachfrage auf das Angebotsmaß zu reduzieren. Nur: die vorgeschlagene regressive Umverteilung von Einkommen verletzte die Interessen der Bevölkerungsmehrheit, die aus Lohnabhängigen zusammengesetzt ist. Sie sagte aber der herrschenden Klasse auch nicht zu, die mit allen Mitteln entschlossen war, den öffentlichen Dienst und die Rolle des Staates in der Wirtschaft zu reduzieren. Im zweiten Akt der Komödie versuchten die heterodoxen Ökonomen an der Macht ihre Rolle herunterzuspielen, indem sie konventionlle Anpassungspolitiken praktizierten, die wenig vom orthodoxen Schema abwichen. Die Chance eines neuen Wachstumszyklus und die Demokratisierung der Wirtschaftspolitik wurden vertan. (Übersetzung aus dem brasilianischen Portugiesisch durch Claudia Preusser) 